\title{
COMPACT SURFACES WITH NO BONNET MATE
}

\author{
GARY R. JENSEN, EMILIO MUSSO, AND LORENZO NICOLODI
}

Abstract. This note gives sufficient conditions (isothermic or totally nonisothermic) for an immersion of a compact surface to have no Bonnet mate.

\section{INTRODUCTION}

Consider a smooth immersion $\mathbf{x}: M \rightarrow \mathbf{R}^{3}$ of a connected, orientable surface $M$, with unit normal vector field $\mathbf{e}_{3}$. Its induced metric $I=d \mathbf{x} \cdot d \mathbf{x}$ and the orientation of $M$ induced by $\mathbf{e}_{3}$ from the standard orientation of $\mathbf{R}^{3}$ induce a complex structure on $M$, which provides a decomposition into bidegrees of the second fundamental form $I I$ of $\mathbf{x}$ relative to $\mathbf{e}_{3}$,

$$
-d \mathbf{e}_{3} \cdot d \mathbf{x}=I I=I I^{2,0}+H I+I I^{0,2} .
$$

Here $H$ is the mean curvature of $\mathbf{x}$ relative to $\mathbf{e}_{3}$ and $I I^{2,0}=\overline{I I^{0,2}}$ is the Hopf quadratic differential of $\mathbf{x}$. Relative to a complex chart $(U, z)$ in $M$,

$$
I=e^{2 u} d z d \bar{z}, \quad I I^{2,0}=\frac{1}{2} h e^{2 u} d z d z,
$$

where the conformal factor $e^{u}$, the Hopf invariant $h$, and the mean curvature $H$ satisfy the structure equations on $U$ relative to $z$,

$$
\begin{aligned}
-4 e^{-2 u} u_{z \bar{z}} & =H^{2}-|h|^{2} \quad \text { Gauss equation } \\
\left(e^{2 u} h\right)_{\bar{z}} & =e^{2 u} H_{z} \quad \text { Codazzi equation }
\end{aligned}
$$

and the Gauss curvature is $K=H^{2}-|h|^{2}$. See [JMN16, page 212].

In 1867 Bonnet Bon67 began an investigation into the problem of whether there exist noncongruent immersions $\mathbf{x}, \tilde{\mathbf{x}}: M \rightarrow \mathbf{R}^{3}$ with the same induced metric, $I=\tilde{I}$, and the same mean curvature, $H=\tilde{H}$. This Bonnet Problem has been studied by Bianchi Bia09, Graustein [Gra24, Cartan [Car42], Lawson-Tribuzy [LT81, Chern Che85, Kamberov-Pedit-Pinkall [KPP98], Bobenko-Eitner [BE98, BE00, Roussos-Hernandez [RH90], Sabitov Sab12, the present authors [JMN16], and many others cited in these references.

Definition 1. An immersion $\mathbf{x}: M \rightarrow \mathbf{R}^{3}$ is Bonnet if there is a noncongruent immersion $\tilde{\mathbf{x}}: M \rightarrow \mathbf{R}^{3}$ such that $\tilde{I}=I$ and $\tilde{H}=H$. Then $\tilde{\mathbf{x}}$ is called a Bonnet mate of $\mathbf{x}$ and $(\mathbf{x}, \tilde{\mathbf{x}})$ form a Bonnet pair.

A constant mean curvature (CMC) immersion $\mathbf{x}: M \rightarrow \mathbf{R}^{3}$, for which $M$ is simply connected and $\mathbf{x}$ is not totally umbilic, admits a 1-parameter family of Bonnet mates, which are known as the associates of $\mathbf{x}$ JMN16, Example 10.11, page

2000 Mathematics Subject Classification. 53C42, 53A10, 53A05.

Authors partially supported by MIUR (Italy) under the PRIN project Varietà reali e complesse: geometria, topologia e analisi armonica; and by the GNSAGA of INdAM. 
302]. The local problem is thus to determine if an immersion $\mathbf{x}$ with nonconstant mean curvature has a Bonnet mate. By nonconstant mean curvature $H$ we mean that $d H \neq 0$ on a dense, open subset of $M$.

Definition 2. A Bonnet immersion $\mathbf{x}: M \rightarrow \mathbf{R}^{3}$ is proper if its mean curvature is nonconstant and there exist at least two noncongruent Bonnet mates.

It is known [JMN16, page 211] that the umbilics of $\mathbf{x}$ are precisely the zeros of its Hopf quadratic differential $I I^{2,0}$. For the following definitions we assume that $\mathbf{x}$ has no umbilics in the domain $U$. If $(U, z)$ is a complex coordinate chart in $M$, then the local coefficient $e^{2 u} h$ of $2 I I^{2,0}$ in $U$ has the polar representation

$$
e^{2 u} h=e^{G+i g},
$$

for a smooth function $G: U \rightarrow \mathbf{R}$ and a smooth map $e^{i g}: U \rightarrow \mathbf{S}^{1}$. The function $g: U \rightarrow \mathbf{R}$ is defined only locally, up to an additive integral multiple of $2 \pi$. If $w=w(z)$ is another complex coordinate in $U$, and if the invariants relative to it are denoted by $\hat{u}$ and $\hat{h}$, then

$$
e^{2 u} h=e^{2 \hat{u}} \hat{h}\left(w^{\prime}\right)^{2},
$$

where $w^{\prime}=\frac{d w}{d z}$ is a nowhere zero holomorphic function of $z$. Setting $e^{2 \hat{u}} \hat{h}=e^{\hat{G}+i \hat{g}}$ on $U$, we find by an elementary calculation

$$
g_{\bar{z} z}=\hat{g}_{\bar{z} z}
$$

on $U$. The Laplace-Beltrami operator of $(M, I)$ is given in the local chart $(U, z)$ by $\Delta=4 e^{-2 u} \frac{\partial^{2}}{\partial z \partial \bar{z}}$. We conclude from (2) that $\Delta g=\Delta \hat{g}$ on $U$, and therefore that $\Delta g$ is a globally defined smooth function on $M$ away from the umbilic points of $\mathbf{x}$.

Definition 3. A surface immersion $\mathbf{x}: M \rightarrow \mathbf{R}^{3}$ is called isothermic if it has an atlas of charts $(U,(x, y))$ each of which satisfies $I=e^{2 u}\left(d x^{2}+d y^{2}\right)$ and $I I=$ $e^{u}\left(a d x^{2}+c d y^{2}\right)$ [JMN16, Definition 9.5, page 277].

Definition 3 is equivalent to the following definition if there are no umbilics [JMN16, Corollary 9.14, page 280].

Definition 4. An umbilic free immersion $\mathbf{x}: M \rightarrow \mathbf{R}^{3}$ of an oriented connected surface is isothermic if $\Delta g=0$ identically on $M$. It is totally nonisothermic if $\Delta g \neq 0$ on a connected, open, dense subset of $M$.

The following is known about umbilic free immersions $\mathbf{x}: M \rightarrow \mathbf{R}^{3}$ for which $M$ is simply connected. Cartan Car42 proved that if $\mathbf{x}$ is proper Bonnet, then it has a 1-parameter family of distinct mates [JMN16, Theorem 10.42, pages 340342]. Graustein [Gra24 proved that if $\mathbf{x}$ is isothermic and Bonnet, then it is proper Bonnet. The present authors [JMN16, Theorem 10.13, pages 303-304] proved that if $\mathbf{x}$ is totally nonisothermic, then it has a unique Bonnet mate.

What is the global situation? In particular, if $M$ is compact, can an immersion $\mathbf{x}: M \rightarrow \mathbf{R}^{3}$ have a Bonnet mate? It is known, and proved in the next section, that a necessary condition that $\mathbf{x}$ be Bonnet is that its set of umbilics is a discrete subset of $M$. Lawson-Tribuzy [LT81] proved that $\mathbf{x}$ cannot be proper Bonnet if $M$ is compact. Roussos-Hernandez [RH90] proved that $\mathbf{x}: M \rightarrow \mathbf{R}^{3}$ has no Bonnet mate if $M$ is compact and $\mathbf{x}$ is a surface of revolution with nonconstant mean curvature. Sabitov [Sab12, Theorem 13, page 144] gives a sufficient condition 
preventing the existence of a Bonnet mate when the mean curvature is nonconstant and $M$ is compact. He gives no geometric interpretation of his condition.

The goal of this paper is to prove the following result. It generalizes the RoussosHernandez result, since a surface of revolution is isothermic [JMN16, Example 9.7, page 277]. It also gives a geometrical clarification of the Sabitov result.

Theorem. Let $\mathbf{x}: M \rightarrow \mathbf{R}^{3}$ be a smooth immersion with nonconstant mean curvature $H$ of a compact, connected surface, and suppose that $\mathcal{D}$, the set of umbilics of $\mathbf{x}$, is a discrete subset of $M$.

(1) If $\mathbf{x}: M \backslash \mathcal{D}$ is isothermic, then $\mathbf{x}$ has no Bonnet mate.

(2) If $\mathbf{x}$ is totally nonisothermic, then it has no Bonnet mate.

\section{The Deformation QuAdratic Differential}

From the Gauss equation above, the Hopf invariants $h$ and $\tilde{h}$ relative to a complex coordinate $z$ of two immersions with the same induced metric and the same mean curvatures must satisfy

$$
|\tilde{h}|=|h|,
$$

since $\tilde{u}=u$. Hence, the only possible difference in the invariants of two such immersions must be in the arguments of the complex valued functions $h$ and $\tilde{h}$. Moreover, taking the difference of their Codazzi equations, we get

$$
\left(e^{2 u} \tilde{h}-e^{2 u} h\right)_{\bar{z}}=e^{2 u}\left(H_{z}-H_{z}\right)=0,
$$

at every point of the domain $U$ of the complex coordinate $z$. This means that the function

$$
F=e^{2 u}(\tilde{h}-h): U \rightarrow \mathbf{C}
$$

is holomorphic.

Definition 5. If $\mathbf{x}, \tilde{\mathbf{x}}: M \rightarrow \mathbf{R}^{3}$ are immersions that induce the same complex structure on $M$, then their deformation quadratic differential is

$$
\mathcal{Q}=\widetilde{I I}^{2,0}-I I^{2,0}
$$

If $\mathbf{x}$ and $\tilde{\mathbf{x}}$ have the same induced metric and mean curvature, then the expression for $\mathcal{Q}$ relative to a complex coordinate $z$ is

$$
\mathcal{Q}=\frac{1}{2} e^{2 u}(\tilde{h}-h) d z d z=\frac{1}{2} F d z d z,
$$

which shows that $\mathcal{Q}$ is a holomorphic quadratic differential on $M$, and

$$
\left|F+e^{2 u} h\right|=\left|e^{2 u} \tilde{h}\right|=\left|e^{2 u} h\right|
$$

on $U$, since $|\tilde{h}|=|h|$. $\mathcal{Q}$ is identically zero on $M$ if and only if $\tilde{h}=h$ in any complex coordinate system. Therefore, by Bonnet's Congruence Theorem, $\mathcal{Q}=0$ if and only if the immersions $\mathbf{x}$ and $\tilde{\mathbf{x}}$ are congruent in the sense that there exists a rigid motion $(\mathbf{y}, A) \in \mathbf{E}(3)$ such that $\tilde{\mathbf{x}}=\mathbf{y}+A \mathbf{x}: M \rightarrow \mathbf{R}^{3}$. Thus, an immersion $\tilde{\mathbf{x}}: M \rightarrow \mathbf{R}^{3}$ is a Bonnet mate of $\mathbf{x}: M \rightarrow \mathbf{R}^{3}$ if it induces the same metric and mean curvature and the deformation quadratic differential is not identically zero.

Proposition 6. If an immersion $\mathbf{x}: M \rightarrow \mathbf{R}^{3}$ possesses a Bonnet mate $\tilde{\mathbf{x}}: M \rightarrow$ $\mathbf{R}^{3}$, then the umbilics of $\mathbf{x}$ must be isolated and coincide with those of $\tilde{\mathbf{x}}$. 
Proof. Under the given assumptions, the holomorphic quadratic differential $\mathcal{Q}$ is not identically zero. Therefore, in any complex coordinate chart $(U, z)$, we have $\mathcal{Q}=\frac{1}{2} F d z d z$, where $F$ is a nonzero holomorphic function of $z$. Its zeros must be isolated. A point $m \in U$ is an umbilic of $\mathbf{x}$ if and only if $h(m)=0$ if and only if $\tilde{h}(m)=0$, by (4). In either case $F(m)=0$ by (4). Therefore, the set of umbilic points is a subset of the set of zeros of $\mathcal{Q}$, which is a discrete subset of $M$.

Let $\mathbf{x}: M \rightarrow \mathbf{R}^{3}$ be an immersion with a Bonnet mate $\tilde{\mathbf{x}}: M \rightarrow \mathbf{R}^{3}$. Let $(U, z)$ be a complex coordinate chart in $M$ and let $h$ and $\tilde{h}$ be the Hopf invariants of $\mathbf{x}$ and $\tilde{\mathbf{x}}$, respectively, relative to $z$ on $U$. Let $\mathcal{D}$ be the set of umbilics of $\mathbf{x}$, necessarily a discrete subset of $M$. On $U \backslash \mathcal{D}$ we have $h$ never zero and

$$
\tilde{h}=h A,
$$

for a smooth function $A: U \backslash \mathcal{D} \rightarrow \mathbf{S}^{1}$, where $\mathbf{S}^{1} \subset \mathbf{C}$ is the unit circle. On $U \backslash \mathcal{D}$ then, the difference of the Hopf differentials is the holomorphic quadratic differential

$$
\mathcal{Q}=\widetilde{I I^{2,0}}-I I^{2,0}=I I^{2,0}(A-1) .
$$

This shows that $A: M \backslash \mathcal{D} \rightarrow \mathbf{S}^{1}$ is a well-defined smooth map on all of $M \backslash \mathcal{D}$.

Remark 7. Under our assumption of nonconstant $H$, the map A cannot be constant, for otherwise $I I^{2,0}$ would then be holomorphic and thus $H$ would be constant by the Codazzi equation.

Proposition 8 (Sabitov[Sab12]). If an immersion $\mathbf{x}: M \rightarrow \mathbf{R}^{3}$ possesses a Bonnet mate $\tilde{\mathbf{x}}: M \rightarrow \mathbf{R}^{3}$, then the deformation quadratic differential $\mathcal{Q}$ of $\mathbf{x}$ is zero only at the umbilics of $\mathbf{x}$. Therefore, $A: M \backslash \mathcal{D} \rightarrow \mathbf{S}^{1}$ never takes the value $1 \in \mathbf{S}^{1}$.

Proof. This is Theorem 1, pages 113ff of Sab12. He says the result is stated in Bob08, but he believes the proof there is inadequate. Sabitov's proof uses results from the Hilbert boundary-value problem. The following proof is essentially the same as Sabitov's, but avoids use of the Hilbert boundary-value problem.

Seeking a contradiction, suppose $\mathcal{Q}\left(m_{0}\right)=0$ for some point $m_{0} \in M \backslash \mathcal{D}$. Since $\mathcal{Q}$ is holomorphic, and not identically zero, its zeros are isolated. Let $(U, z)$ be a complex coordinate chart of $M \backslash \mathcal{D}$ centered at $m_{0}$, containing no other zeros of $\mathcal{Q}$, and such that $z(U)$ is an open disk of $\mathbf{C}$. Now $A\left(m_{0}\right)=1$ and $A$ is continuous, so we may assume $U$ chosen small enough that $A$ never takes the value -1 on $U$. Then there exists a smooth map $v: U \rightarrow \mathbf{R}$ such that $-\pi<v<\pi$ and $A=e^{i v}$ on $U$. Since $A=1$ on $U$ only at $m_{0}$, it follows that

$$
v\left(U \backslash\left\{m_{0}\right\}\right) \subset(-\pi, 0) \text { or } v\left(U \backslash\left\{m_{0}\right\}\right) \subset(0, \pi) .
$$

Let $e^{2 u}$ and $h$ be the conformal factor and Hopf invariant of $\mathbf{x}$ relative to $z$. Then $h$ never zero on $U$ implies it has a polar representation $h=e^{f+i g}$, for some smooth functions $f, g: U \rightarrow \mathbf{R}$. Now $\mathcal{Q}=\frac{1}{2} F d z d z$, where

$$
F=e^{2 u} e^{f+i g}\left(e^{i v}-1\right)=e^{2 u+f}\left(e^{i(g+v)}-e^{i g}\right): U \rightarrow \mathbf{C}
$$

is holomorphic. Using the identity

$$
e^{i(g+v)}-e^{i g}=e^{i(2 g+v) / 2}\left(e^{i v / 2}-e^{-i v / 2}\right)=2 i e^{i(g+v / 2)} \sin (v / 2),
$$

we get

$$
F=2 i e^{2 u+f+i(g+v / 2)} \sin (v / 2)
$$


on $U$. The contour integral of $d \log F$ about any circle in $U$ centered at $m_{0}$ is $2 \pi i$ times the number of zeros of $F$ inside the circle. By assumption, this integral is not zero. But,

$$
d \log F=d(2 u+f+i(g+v / 2))+d \log (|\sin (v / 2)|),
$$

and the contour integral of the right hand side is zero, since these are exact differentials on $U \backslash\left\{m_{0}\right\}$. In fact, the values of $v / 2$ on $U \backslash\left\{m_{0}\right\}$ lie entirely in $(0, \pi / 2)$ or entirely in $(-\pi / 2,0)$, so $\sin (v / 2)$ is never zero. This is the desired contradiction to our assumption that $\mathcal{Q}$ has a zero in $M \backslash \mathcal{D}$.

As a consequence of this Proposition, the smooth map $A: M \backslash \mathcal{D} \rightarrow \mathbf{S}^{1}$ never takes the value $1 \in \mathbf{S}^{1}$, so there exists a smooth map

$$
r: M \backslash \mathcal{D} \rightarrow(0,2 \pi) \subset \mathbf{R},
$$

such that $A=e^{i r}$ on $M \backslash \mathcal{D}$.

\section{Proof of the Theorem}

Proof. Seeking a contradiction, we suppose that $\mathbf{x}$ possesses a Bonnet mate $\tilde{\mathbf{x}}$ : $M \rightarrow \mathbf{R}^{3}$. Let $I I^{2,0}$ and $\widetilde{I I^{2,0}}$ be the Hopf quadratic differentials of $\mathbf{x}$ and $\tilde{\mathbf{x}}$, respectively. By the preceding propositions, the quadratic differential $\widetilde{I I^{2,0}}-I I^{2,0}$ is holomorphic on $M$, and on $M \backslash \mathcal{D}$

$$
\widetilde{I I^{2,0}}-I I^{2,0}=I I^{2,0}\left(e^{i r}-1\right),
$$

where the function $r: M \backslash \mathcal{D} \rightarrow(0,2 \pi)$ is smooth. Let $(U, z)$ be a complex coordinate chart in $M \backslash \mathcal{D}$. Let $h$ and $e^{u}$ be the Hopf invariant and conformal factor of x relative to $z$. Then $h=e^{f+i g}$ on $U$, for some smooth functions $f: U \rightarrow \mathbf{R}$ and $e^{i g}: U \rightarrow \mathbf{S}^{1}$.

1). If $\mathbf{x}$ is isothermic, then $g_{\bar{z} z}=0$ identically on $U$. Let $G=f+2 u: U \rightarrow \mathbf{R}$. Then $\left(e^{G+i g}\left(e^{i r}-1\right)\right)_{\bar{z}}=0$ implies

$$
r_{\bar{z}}=i(G+i g)_{\bar{z}}\left(1-e^{-i r}\right)
$$

on $U$. Applying $\partial_{z}$ to this, and using that $r_{z}$ is the complex conjugate of $r_{\bar{z}}$, we find

$$
r_{\bar{z} z}=0
$$

on $U$. Hence, $r: M \backslash \mathcal{D} \rightarrow(0,2 \pi)$ is a bounded harmonic function. Since the points of $\mathcal{D}$ are isolated and $r$ is bounded, we know that $r$ extends to a harmonic function on all of $M$. But then $r$ must be constant, since $M$ is compact. This contradicts our assumption of nonconstant $H$, by Remark 7

2). If $\mathrm{x}$ is totally nonisothermic, we have either $\Delta g \leq 0$ or $\Delta g \geq 0$ on $M \backslash \mathcal{D}$. To be specific, let us suppose that $\Delta g \leq 0$ on $M \backslash \mathcal{D}$. Now (6) holds and by the proof of Theorem 10.13 on pages 303-304 of [JMN16, we have

$$
e^{i r}=1+\frac{-2 g_{\bar{z} z}}{D}\left(g_{\bar{z} z}+i L\right),
$$

on $U$, where $L=\left|G_{\bar{z}}+i g_{\bar{z}}\right|^{2}-G_{\bar{z} z}$ and $D=g_{\bar{z} z}^{2}+L^{2}$. Applying $\partial_{z}$ to (6) and using (8), we find

$$
r_{\bar{z} z}=-2 g_{\bar{z} z},
$$

on $U$. Therefore, $\Delta r=-2 \Delta g \geq 0$ on $M \backslash \mathcal{D}$. 
Recall [HK76, Def. $\S 2.1$, pages 40-41] that a function $v: V \rightarrow \mathbf{R} \cup\{-\infty\}$ on a domain $V \subset \mathbf{C}$ is subharmonic if

(1) $-\infty \leq v(z)<+\infty$ in $V$.

(2) $v$ is upper semi-continuous in $V$. (This means that for any $c \in \mathbf{R}$, the set $\{z \in U: v(z)<c\}$ is open in $\mathrm{V}$.)

(3) If $z_{0}$ is any point of $V$ then there exist arbitrarily small positive values of $R$ such that

$$
v\left(z_{0}\right) \leq \frac{1}{2 \pi R} \int_{0}^{2 \pi} v\left(z_{0}+R e^{i t}\right) d t
$$

If $v$ is of class $C^{2}$ in $V$, then $v$ is subharmonic in $V$ if and only if $v_{\bar{z} z} \geq 0$ in $V$ [HK76, Example 3, page 41].

If $M$ is a connected Riemann surface, we define a function $v: M \rightarrow \mathbf{R} \cup\{-\infty\}$ to be subharmonic if for any complex coordinate chart $(U, z)$ of $M$, the local representative $v \circ z^{-1}: z(U) \rightarrow \mathbf{R}$ is subharmonic. This is well-defined by the Corollary to Theorem 2.8 on page 53 of [HK76].

We conclude from (9) that $r$ is subharmonic on $M \backslash \mathcal{D}$. In the event that $\Delta g \geq 0$ on $M \backslash \mathcal{D}$, we conclude that $-r$ is subharmonic and continue as below with $-r$.

Suppose $(U, z)$ is a complex coordinate chart centered at a point $m_{0} \in \mathcal{D}$, and small enough that no other point of $\mathcal{D}$ lies in it. Then $r \circ z^{-1}$ is subharmonic on the open set $z(U) \backslash\{0\}$, so it extends uniquely to a subharmonic function on $z(U)$, by Theorem 5.8 on page 237 of [HK76]. It follows that $r$ extends uniquely to a subharmonic function on $M$.

By Theorem 1.2 on page 4 of [HK76], if $v: V \rightarrow \mathbf{R} \cup\{-\infty\}$ is upper semicontinuous on a nonempty compact domain $V \subset \mathbf{C}$, then $v$ attains its maximum on $V$; i.e., there exists $z_{0} \in V$ such that $v(z) \leq v\left(z_{0}\right)$ for all $z \in V$. The same proof shows that this is true for an upper semi-continuous function on a compact Riemann surface. Thus, the subharmonic function $r: M \rightarrow \mathbf{R} \cup\{-\infty\}$ attains its maximum at some point $m_{0} \in M$. Let $(U, z)$ be a complex coordinate chart centered at $m_{0}$. Choose $R>0$ such that the disk $D(0, R)=\{z \in \mathbf{C}:|z| \leq R\}$ is contained in $z(U)$. By the maximum principle for subharmonic functions [HK76, Theorem 2.3, page 47], $r \circ z^{-1}$ must be constantly equal to $r\left(m_{0}\right)$ on $D(0, R)$. It follows that

$$
E=\left\{m \in M: r(m)=r\left(m_{0}\right)\right\}
$$

is an open subset of $M$. But

$$
E=M \backslash\left\{m \in M: r(m)<r\left(m_{0}\right)\right\}
$$

is closed, since $r$ is upper semi-continuous. We conclude that $r$ is constant on $M$, which is our sought for contradiction, by Remark 7.

\section{REFERENCES}

[BE98] Alexander Bobenko and Ulrich Eitner. Bonnet surfaces and Painlevé equations. J. Reine Angew. Math., 499:47-79, 1998.

[BE00] Alexander I. Bobenko and Ulrich Eitner. Painlevé equations in the differential geometry of surfaces, volume 1753 of Lecture Notes in Mathematics. Springer-Verlag, Berlin, 2000.

[Bia09] Luigi Bianchi. Lezioni di geometria differenziale, volume 1-3. E. Spoerri, Pisa, 19031909. Seconda edizione riveduta e considerevolmente aumentata. 
[Bob08] Alexander I. Bobenko. Exploring surfaces through methods from the theory of integrable systems: the Bonnet problem. In Surveys on geometry and integrable systems, volume 51 of Adv. Stud. Pure Math., pages 1-53. Math. Soc. Japan, Tokyo, 2008.

[Bon67] Pierre Ossian Bonnet. Mémoire sur la théorie des surfaces applicables sur une surface donnée, deuxième partie. Journal de l'Ecole Polytechnique, 42:1 - 151, 1867.

[Car42] Elie Cartan. Sur les couples de surfaces applicables avec conservation des courbures principales. Bull. Sc. Math., 66:55-85, 1942. Oeuvres Complète, Partie III, Volume 2, pp. 1591-1620.

[Che85] Shiing-Shen Chern. Deformation of surfaces preserving principal curvatures. In I. Chavel and H.M. Farkas, editors, Differential Geometry and Complex Analysis: A Volume Dedicated to the Memory of Harry Ernest Rauch, pages 155 - 163, Berlin, 1985. SpringerVerlag.

[Gra24] W. C. Graustein. Applicability with preservation of both curvatures. Bull. Amer. Math. Soc., 30(1-2):19-23, 1924.

[HK76] W. K. Hayman and P. B. Kennedy. Subharmonic functions. Vol. I. Academic Press [Harcourt Brace Jovanovich, Publishers], London-New York, 1976. London Mathematical Society Monographs, No. 9.

[JMN16] Gary R. Jensen, Emilio Musso, and Lorenzo Nicolodi. Surfaces in classical geometries. Universitext. Springer, Cham, 2016. A treatment by moving frames.

[KPP98] George Kamberov, Franz Pedit, and Ulrich Pinkall. Bonnet pairs and isothermic surfaces. Duke Math. J., 92(3):637-644, 1998.

[LT81] H. Blaine Lawson, Jr. and Renato de Azevedo Tribuzy. On the mean curvature function for compact surfaces. J. Differential Geom., 16(2):179-183, 1981.

[RH90] Ioannis M. Roussos and Gastón E. Hernández. On the number of distinct isometric immersions of a Riemannian surface into $\mathbf{R}^{3}$ with given mean curvature. Amer. J. Math., 112(1):71-85, 1990.

[Sab12] I. Kh. Sabitov. Isometric surfaces with common mean curvature and the problem of Bonnet pairs. Sbornik: Mathematics, 203(1):111-152, 2012.

(G. R. Jensen) Department of Mathematics, Washington University, One Brookings Drive, St. Louis, MO 63130, USA

E-mail address: gary@wustl.edu

(E. Musso) Dipartimento di Scienze Matematiche, Politecnico di Torino, Corso Duca DEgli Abruzzi 24, I-10129 Torino, ItALy

E-mail address: emilio.musso@polito.it

(L. Nicolodi) Dipartimento di Scienze Matematiche, Fisiche e Informatiche, Università di Parma, Parco Area delle Scienze 53/A, I-43124 Parma, Italy

E-mail address: lorenzo.nicolodi@unipr.it 